Orthopäde $2021 \cdot 50: 237-243$

https://doi.org/10.1007/s00132-020-03936-2

Online publiziert: 25. Juni 2020

(c) Der/die Autor(en) 2020

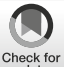

Sebastian Klingebiel' • Sebastian Mühl' ${ }^{2}$ Georg Gosheger' •

Wolfgang Hartmann ${ }^{2}$. Kristian Nikolaus Schneider' - Tymoteusz Borys Budny' Carolin Rickert ${ }^{1}$ - Dominik Schorn ${ }^{1} \cdot$ Niklas Deventer $^{1} \cdot$ Timo Lübben $^{1}$

${ }^{1}$ Klinik für Allgemeine Orthopädie und Tumororthopädie, Universitätsklinikum Münster, Münster, Deutschland

${ }^{2}$ Gerhard-Domagk-Institut für Pathologie, Universitätsklinikum Münster, Münster, Deutschland

\title{
Stabilitätsgefährdende Osteolyse der Tibia durch einen intraossären tenosynovialen Riesenzelltumor
}

\section{Eine außergewöhnliche klinisch- histopathologische Konstellation}

\section{Video online}

Die Online-Version dieses Beitrags (https:// doi.org/10.1007/s00132-020-03936-2) enthält ein Video zur Demonstration des Gangbildes mit angelegter Exoprothese. Beitrag und Video stehen Ihnen auf www. springermedizin.de zur Verfügung. Bitte geben Sie dort den Beitragstitel in die Suche ein, das Zusatzmaterial finden Sie beim Beitrag unter „Ergänzende Inhalte“.

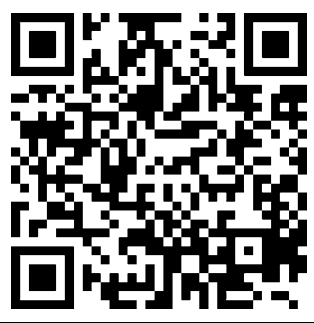

Wir berichten über den seltenen klinischen Fall eines unterschenkelamputierten Patienten mit stabilitätsgefährdender Osteolyse der proximalen Tibia durch eine intraossäre Rezidivmanifestation seitens eines tenosynovialen Riesenzelltumors (TSGCT; vormals: pigmentierte

Die vorliegende Arbeit ist in der Klinik für Orthopädie und Tumororthopädie des Universitätsklinikums Münster angefertigt worden. villonoduläre Synovitis). Die Unterschenkelamputation wurde infolge eines lokal nicht beherrschbaren tenosynovialen Riesenzelltumors des oberen Sprunggelenkes durchgeführt. Die intraossäre Manifestation eines TSGCT stellt in diesem Fall eine absolute Rarität und Besonderheit dar.

\section{Anamnese}

Ein 65-jähriger unterschenkelamputierter Patient stellte sich mit einer großen und stabilitätsgefährdenden Osteolyse der proximalen Tibia seines Unterschenkelstumpfes in unserer Sprechstunde vor (• Abb. 1). Er wurde 14 Jahre zuvor aufgrund eines lokal nicht beherrschbaren und rezidivierenden tenosynovialen Riesenzelltumors (TSGCT) des rechten oberen Sprunggelenkes aufgrund exulzerierender extraartikulärer Beteiligung oberhalb der Malleolengabel nach multiplen operativen Revisionen mit einer radikalen Resektion i.S. einer Unterschenkelamputation nach Burgess versorgt. Entsprechend lag damals postoperativ ein R0-Resektionstatus vor. Aufgrund aktuell zunehmender Belastungsschmerzen des Stumpfes in den vergangenen Monaten wurde das bisher komplikationslose Tragen seiner Exoprothese nicht mehr toleriert und der Patient beklagte die hieraus resultierende Immobilität und den drohenden Verlust seiner Autonomie.

\section{Klinischer und radiologischer Befund}

Im Rahmen der klinischen Untersuchung zeigte sich ein äußerlich reizloser Unterschenkelstumpf ohne Infektstigmata oder palpable Resistenzen. Das Kniegelenk war stabil und frei beweglich. Es bestand ein Druckschmerz über der Facies lateralis der Tibia im mittleren Stumpf-

\begin{tabular}{|ll}
\hline Abkürzungen \\
\hline CD & $\begin{array}{l}\text { "Cluster of differentiation" } \\
\text { (immunphänotypische Oberflä- } \\
\text { chenmerkmale von Zellen) }\end{array}$ \\
\hline CSF & "Colony stimulating factor" \\
\hline HE & $\begin{array}{l}\text { Hämatoxylin-Eosin (Färbeverfah- } \\
\text { ren in der Histologie) }\end{array}$ \\
\hline PMMA & $\begin{array}{l}\text { Polymethylmethacrylat (Syntheti- } \\
\text { scher Knochenzement) }\end{array}$ \\
\hline STIR & $\begin{array}{l}\text { "Short tau inversion recovery" } \\
\text { (MRT-Sequenz) }\end{array}$ \\
\hline TSGCT & \begin{tabular}{l} 
Tenosynovialer Riesenzelltumor \\
\hline
\end{tabular}
\end{tabular}




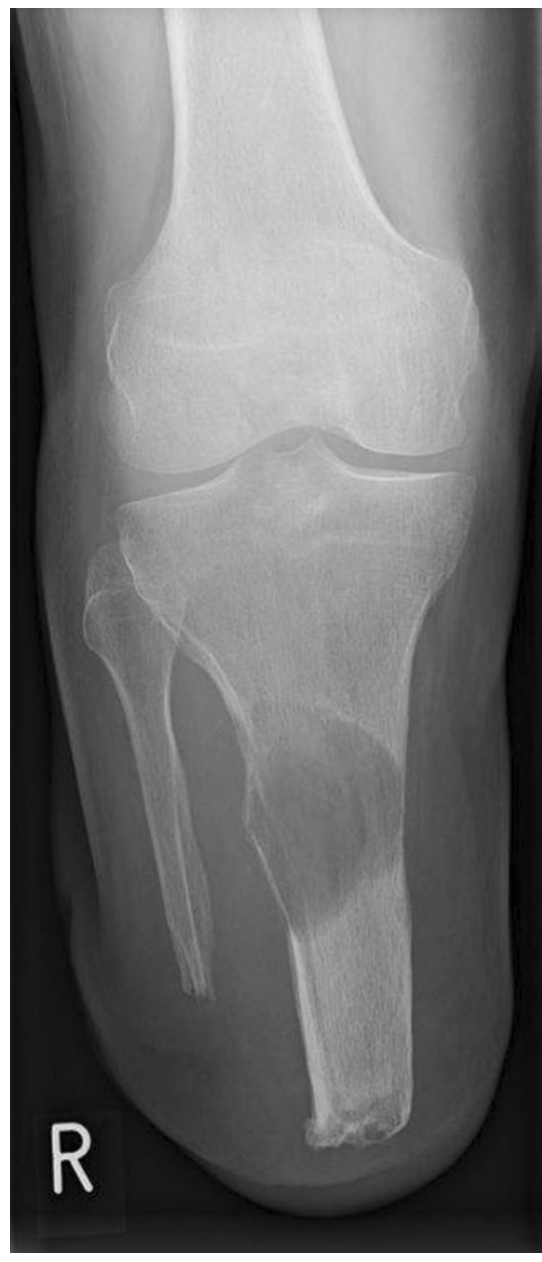

Abb. 1 A Projektionsradiographie in der a.-p.-Ansicht mit stabilitätsgefährdender Osteolyse der proximalen Tibia bei initialer ambulanter Vorstellung und schmerzbedingt deutlich reduzierter Belastungsfähigkeit des Unterschenkelstumpfes

drittel. Auf der bereits vorliegenden nativradiologischen Aufnahme konnte eine große osteolytische Raumforderung der proximalen Tibia mit einer Ausdehnung von etwa $5,5 \times 4 \mathrm{~cm}$ und teilweise fehlender Respektierung der Kortikalis nachgewiesen werden - entsprechend einem Befund Typ 1C nach Lodwick (- Abb. 1). Die nachfolgende MRT-Diagnostik bestätigte den Verdacht einer lokal aggressiven ossären Manifestation mit kleiner extraossärer Weichteilkomponente (•Abb. 2).

\section{Diagnose}

In Zusammenschau stellten wir die dringliche Indikation zur offenen Biopsie. Die konsekutive histopathologische Unter-

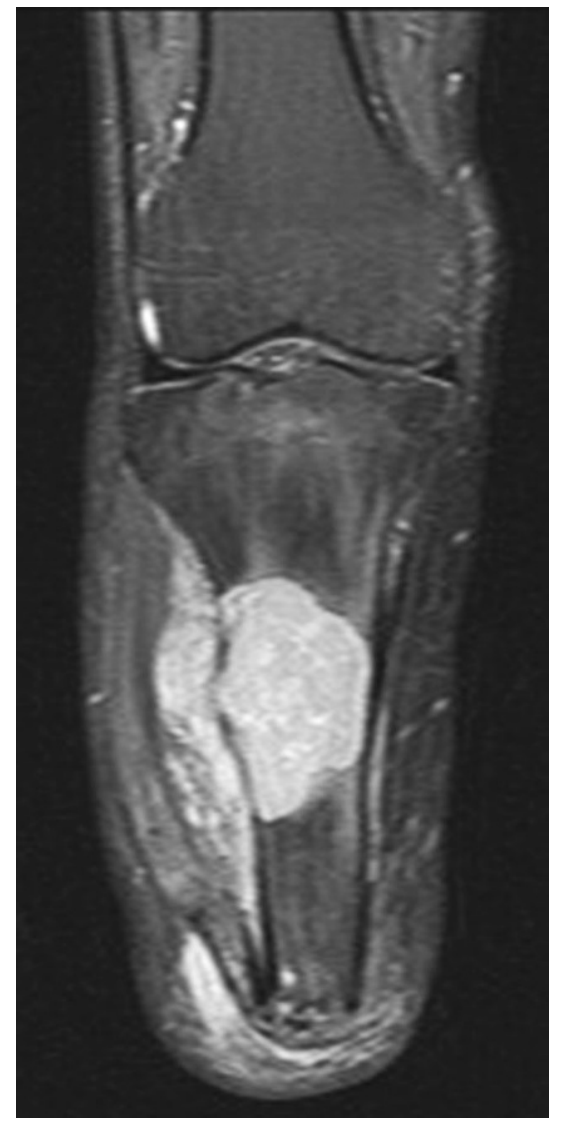

Abb. 2 ॥ DieMRT (koronare Ansicht, STIR[,,short tau inversion recovery"]-Sequenz mit Kontrastmittelapplikation) zeigt eine Kontrastmittelaufnahme der intraossären Läsion des Tibiastumpfes

suchung zeigte eine in größeren Teilen CD68- und CD163-positive mononukleäre Proliferation unter Einschluss mehrkerniger Riesenzellen bei Nachweis ausgedehnter Siderinpigmentablagerungen ohne belegbare atypische Mitosen oder Gewebsnekrosen (• Abb. 3 und 4). Die Next-Generation-Sequencing-Analyse (NGS) auf DNA-Ebene konnte eine H3F3A-Mutation, wie für einen Riesenzelltumor des Knochens typisch, ausschließen. Mittels RNA-Sequenzierung konnte eine Translokation des CSF1Genlokus nachgewiesen werden, wie sie für tenosynoviale Riesenzelltumoren bekannt ist. Unter Berücksichtigung der Gesamtkonstellation wurde die Läsion als intraossäre Manifestation eines tenosynovialen Riesenzelltumors vom diffusen Subtyp eingeordnet.

\section{Therapie und Verlauf}

Neben der Indikation zur marginalen Resektion mittels Kürettage diskutierten wir verschiedene Verfahren zur Stabilisierung der proximalen Tibia. Wir entschieden uns zur Durchführung einer intramedullären Verbundosteosynthese mit zwei kanülierten $6,5 \times 125 \mathrm{~mm}$ Schrauben (Qwix, Fa. Depuy Synthes, Warsaw, IN, USA) und PMMA-Knochenzement (Palacos, Fa. Heraeus medical, Hanau, Deutschland).

Die Wunde heilte per primam, sodass der Patient am 10. postoperativen Tag bereits erstmals unter Anlage seiner Exoprothese für einige Schritte schmerzfrei mobilisierbar war. Die postoperative Röntgenkontrolle zeigte eine suffiziente Kürettage des TSGCT mit regelrechter Lage der Verbundosteosynthese (- Abb.5). Die postoperative histopathologische Untersuchung des Resektates bestätigte die o.g. Diagnose bei R1-Resektionsstatus.

Eine planmäßige Wiedervorstellung zur klinisch-radiologischen Verlaufskontrolle erfolgte 3 Monate postoperativ. Nativradiologisch und MR-graphisch zeigte sich ein konstanter Befund ohne Hinweis auf ein lokales Tumorrezidiv (-Abb.6). Eine pulmonale Manifestation wurde aufgrund der aggressiven Biologie des hier beschriebenen TSGCT als Einzelfallentscheidung CT-graphisch ausgeschlossen. Der Patient berichtete im Rahmen dieser Konsultation bereits über eine vollständige Remobilisation mit unlimitierter Gehstrecke. Er konnte seinen Alltag wieder ohne Einschränkungen bewältigen. Die Narbe war reizlos verheilt und der Stumpf regelrecht konfiguriert (• Abb. 7). Der Patient demonstrierte mit angelegter Exoprothese (- Abb. 8) ein flüssiges und weitestgehend physiologisches Gangbild (siehe Video online zu diesem Beitrag).

Wir haben dem Patienten aufgrund des relativ hohen Risikos für ein Lokalrezidiv weiterhin engmaschige klinisch-radiologische Verlaufskontrollen im 3-monatigen Intervall für zunächst 2 Jahre postoperativ mit lokaler kontrastmittel- 
gestützter MRT-Bildgebung und Projektionsradiographie empfohlen. Anschließend sollten bis einschließlich des 5. postoperativen Jahrs halbjährliche Kontrollen erfolgen.

\section{Diskussion}

Der TSGCT ist mit einer geschätzten Inzidenz von 1,5-2/1.000.000 eine seltene gutartige, aber potenziell lokal aggressive Erkrankung der Gelenkschleimhaut des überwiegend jungen Patienten zwischen 20 und 40 Jahren [7]. Der klinisch-ätiologische Hintergrund von TSGCT ist weitestgehend unklar. Einige Autoren sehen gar in über $50 \%$ der Fälle einen Zusammenhang mit traumatischen Ereignissen [6]. Andere Autoren sehen eine inflammatorische Genese vordergründig [1]. Es wird unterschieden zwischen der lokalen und der diffusen Form des TSGCT. Histologisch sind diese Typen nicht zu unterscheiden. Erstere Form ist klinisch gut umschrieben und kann sich intra- sowie extraartikulär manifestieren. Insbesondere die diffuse Form des TSGCT kann sich lokal aggressiv und mit infiltrativem Wachstumsmuster ausbreiten und manifestiert sich klinisch in erster Linie durch eine schmerzhafte Gelenkschwellung [9, 11]. Genetisch konnte in TSGCT als rekurrentes Ereignis in einer Subpopulation von Zellen eine Translokation mit Beteiligung des CSF1-Genlokus nachgewiesen werden, die über eine CSF1-Überexpression zur Rekrutierung von Makrophagen führt [10]. Prädilektionsstellen dieser auch durch Hämosiderineinlagerungen charakterisierten, zumeist synovialen Läsionen sind große Gelenke wie Knie-, Sprung- und Hüftgelenk (v. a. diffuser Typ), aber auch Sehnenscheiden und Schleimbeutel (eher lokaler Typ) [6] Trotz sorgfältiger arthroskopischer oder offener Resektion sind Lokalrezidive keine Seltenheit [2]. Bei der diffusen Form sind Rezidivraten von bis zu $40 \%$ beschrieben [5]. Daher stellen TSGCT eine besondere diagnostische und therapeutische Herausforderung in der Orthopädie und Rheumatologie dar. Schwerwiegende Verläufe mit lokal nicht beherrschbarem Wachstum und maligner Transformation oder ungewöhnlichen Lokalisationen wie dem Temporomandibular-

Orthopäde 2021 · 50:237-243 https://doi.org/10.1007/s00132-020-03936-2

(c) Der/die Autor(en) 2020

S. Klingebiel · S. Mühl · G. Gosheger · W. Hartmann · K. N. Schneider · T. B. Budny · C. Rickert • D. Schorn · N. Deventer $\cdot$ T. Lübben

Stabilitätsgefährdende Osteolyse der Tibia durch einen
intraossären tenosynovialen Riesenzelltumor. Eine
außergewöhnliche klinisch-histopathologische Konstellation

\section{Zusammenfassung}

Der tenosynoviale Riesenzelltumor (TSGCT) ist eine seltene gutartige Erkrankung von Gelenkschleimhäuten, Sehnenscheiden und Schleimbeuteln. Wir berichten über die ungewöhnliche Konstellation einer intraossären Manifestation der proximalen Tibia eines Unterschenkelstumpfes nach Burgess-Amputation infolge eines lokal nicht beherrschbaren tenosynovialen Riesenzelltumors des oberen Sprunggelenkes.
Die Kürettage des Lokalbefundes und die operative Stabilisierung durch eine intramedulläre Verbundosteosynthese führten zu einer frühzeitigen Rehabilitation der Exoprothesenversorgung mit Wiedererlangung der Patientenautonomie.

Schlüsselwörter

Amputationsstumpf · Osteosynthese . Osteolyse PVNS · Verbundosteosynthese

\section{Imminent fracture of the tibia due to an osteolytic lesion caused by an intraosseous tenosynovial giant cell tumor. An unusual clinico-histopathological constellation}

\section{Abstract}

The tenosynovial giant cell tumor is a rare disease of the joint mucosa, tendon sheaths and bursa. We report on the rare constellation of an intraosseous manifestation of the proximal tibia of a lower leg stump after Burgess amputation as a result of a locally uncontrollable tenosynovial giant cell tumor of the upper ankle. The curettage of the local findings and operative stabilization through an intramedullary composite osteosynthesis led to an early rehabilitation of the exoprosthesis care with regaining patient autonomy.

\section{Keywords}

Amputation stump · Composite osteosynthesis · Exoprosthesis - Osteolysis . TSGCT gelenk sind Ausnahmen, jedoch in der Literatur beschrieben $[4,8]$. Der hier präsentierte Fall, bei dem etliche Jahre zuvor eine lokale Tumorkontrolle des TSGCT nur durch eine radikale Resektion im Sinne einer Unterschenkelamputation mit R0-Status herbeigeführt werden konnte, stellt eine absolute Rarität dar. Eine exzeptionelle Konstellation ist bei dieser Rezidivmanifestation die nahezu ausschließlich intraossäre Lokalisation des TSGCT. Die Manifestation des TSGCT im Knochen, offenbar als Sekundärmanifestation, zeigt in diesem Einzelfall das für einen TSGCT ungewöhnliche aggressive biologische Potenzial des Prozesses. Dass dieser Befund dennoch kein Einzelfall ist, zeigen aktuelle Fallberichte mit Beschreibung eines TSGCT im Os sacrum und Entwicklung neurologischer Symptomatik, sowie ein weiterer Fall mit
Infiltration von Azetabulum und Oberschenkelhals bei TSGCT des Hüftgelenkes [3]. Dass es sich in unserem Fallbeispiel bei dem TSGCT des Amputationsstumpfes um ein Spätrezidiv handelt, ist zwar wahrscheinlich, schlussendlich jedoch nicht sicher nachvollziehbar. Die zeitliche Latenz von 14 Jahren bis zur Manifestation eines sehr späten Rezidivs und das offensichtlich in diesem Fall diskontinuierliche Wachstum sind hierbei eine weitere außergewöhnliche Konstellation. Bei der Literaturrecherche ließ sich eine vergleichbare Konstellation nach R0Reektion nicht identifizieren.

Das erfreuliche postoperative Ergebnis der oben dargestellten unkonventionellen Verbundosteosynthese, die dem Patienten die Wiedererlangung seiner Mobilität gewährte, ist in diesem Fall ebenfalls hervorzuheben. Weitere 


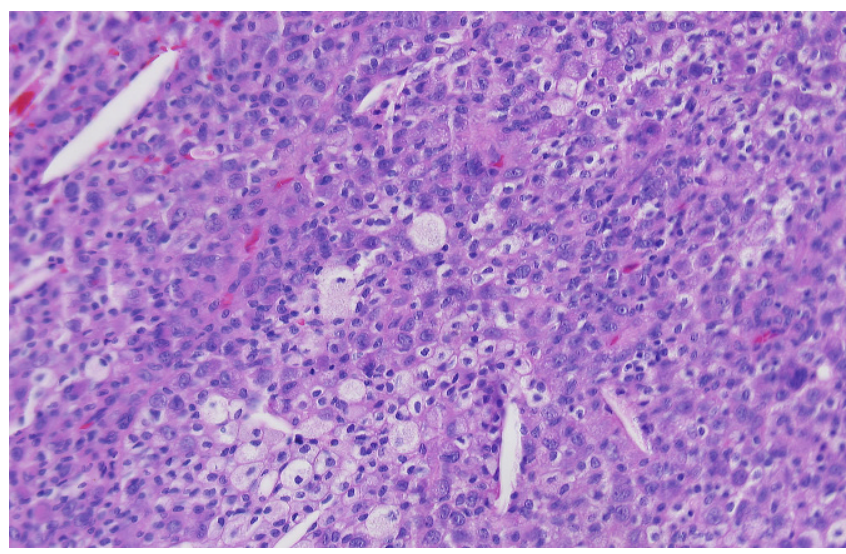

Abb. 3 ॥ Histologischer Befund in Hämatoxylin-Eosin(HE)-Färbung: Proliferation von mononukleären synovialen Zellen, mehrkernigen Riesenzellen, lymphozellulären Infiltraten und Depots von Eisenpigmenten

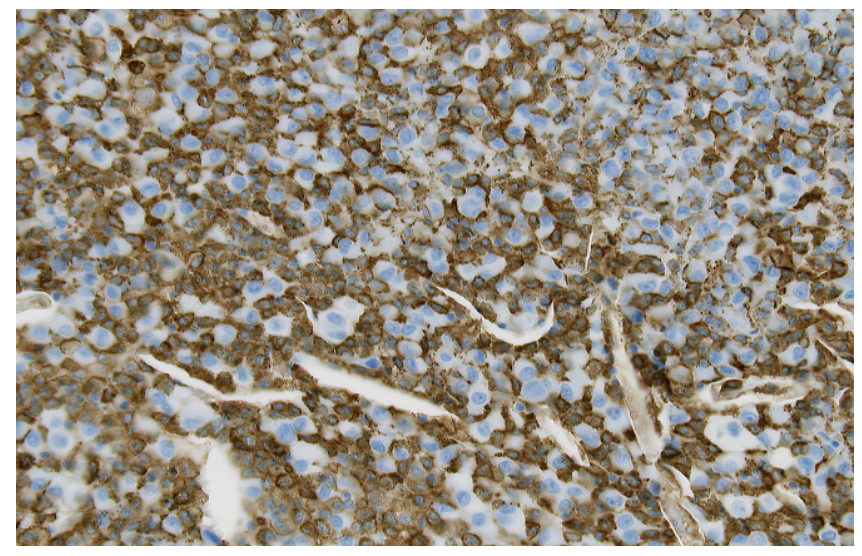

Abb. $4 \Delta$ Histologischer Befund in CD163-Färbung mit Darstellung von Makrophagen/Monozyten als repräsentative biologische Marker für tenosynoviale Riesenzelltumoren. Mittels RNA-Sequenzierung konnte eine Translokation des CSF1 ("colony stimulating factor 1")-Genlokus nachgewiesen werden, wie sie für tenosynoviale Riesenzelltumoren bekannt ist

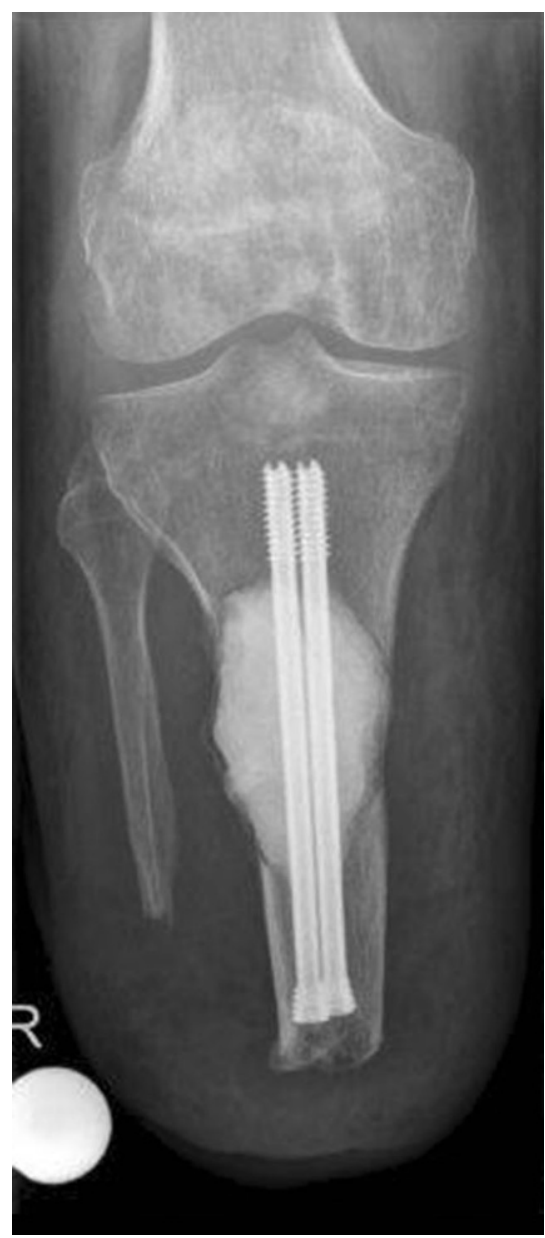

Abb. 5 A Postoperative a. p.-Projektionsradiographie des Kniegelenkes mit Abbildung der proximalen Tibia nach Kürettage des Lokalbefundes und Verbundosteosynthese mit zwei kanülierten Schrauben $(6,5 \times 125 \mathrm{~mm}$, Qwix, Fa. Depuy Synthes) und Polymethylmethacrylat(PMMA)-Knochenzement (Palacos, Fa. Heraeus medical)

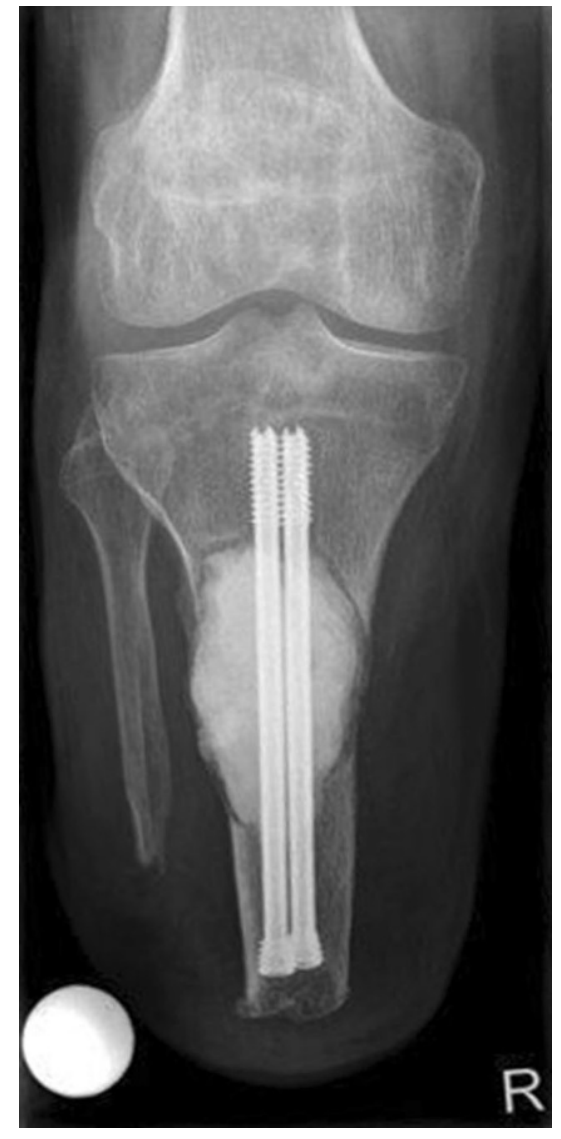

Abb. 6 ム Projektionsradiographische Verlaufskontrolle des Kniegelenkes mit Darstellung der proximalen Tibia in a.p.-Projektion 3 Monate postoperativ: Weitestgehend konstanter radiologischer Lokalbefund ohne Tumorprogress/-persistiv oder Materialversagen engmaschige klinisch-radiologische Verlaufskontrollen werden zeigen, ob das bisherige klinische Ergebnis bestätigt werden kann. Durch die Art der Versorgung mit der intramedullären Verbundosteosynthese konnte zum einen das Kniegelenk als funktionell entscheidendes Element der suffizienten und bisher seitens des Patienten gut angenommenen Exoprothesenversorgung erhalten werden. Die Verwendung des PMMAZementes hat neben der Augmentation der Läsion auch den Aspekt der Adjuvanz im Sinne einer Thermodesinfektion im Rahmen der exothermen Reaktion bei Aushärtung. Dies kann der lokalen Tumorkontrolle dienen. Zum anderen konnte hierdurch eine auftragende Plattenosteosynthese der proximalen Tibia unterbleiben. Diese hätte möglicherweise für Druckschäden im Weichgewebe oder Irritationen der Liner- und Prothesenanpassung gesorgt, sodass eine dauerhafte adäquate Exoprothesenversorgung gefährdet gewesen wäre. In der Literatur konnte eine vergleichbare Stumpfversorgung nicht gefunden werden, wodurch der experimentelle Charakter dieser operativen Therapie unterstrichen wird.

\section{Fazit für die Praxis}

\section{- Ein TSGCT (tenosynovialer Riesen- zelltumor) kann sich grundsätzlich intraossär als osteolytische Läsion}




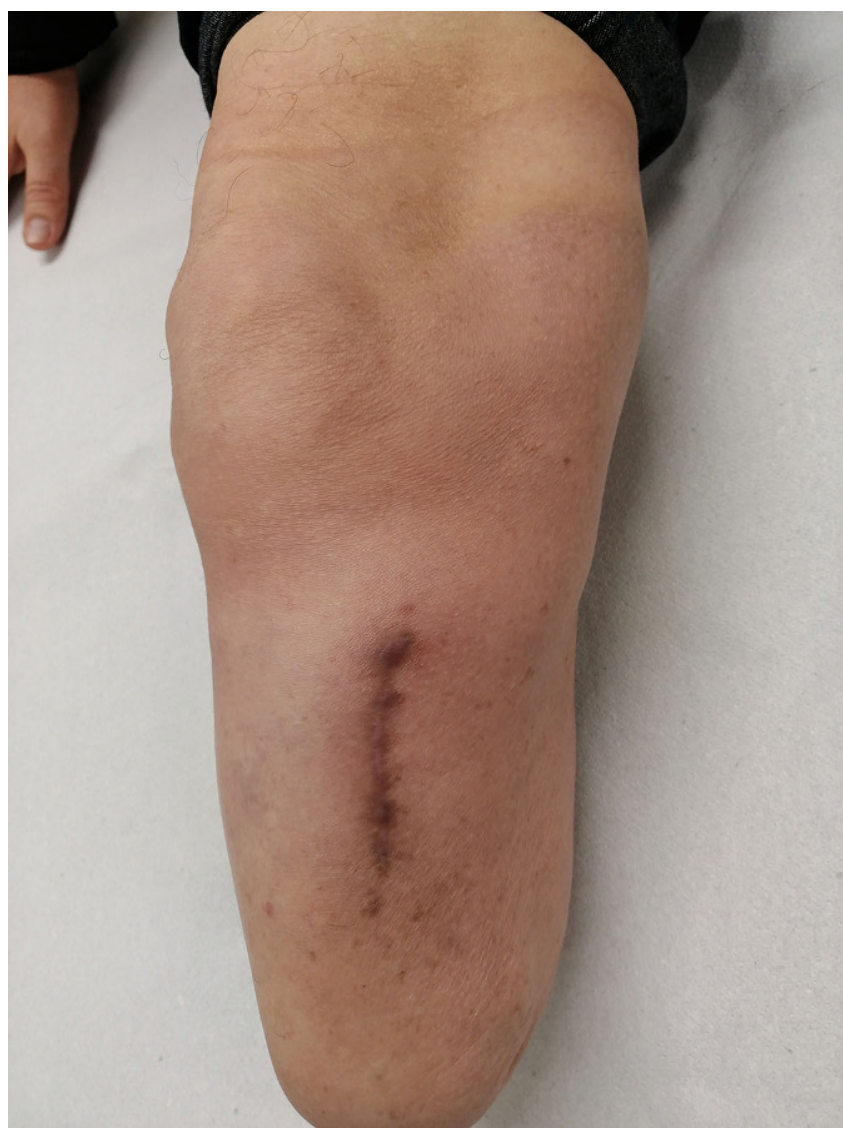

Abb. 7 A Klinisches Bild des reizlosen Stumpfes 3 Monate postoperativ mit vollständiger Beschwerdefreiheit

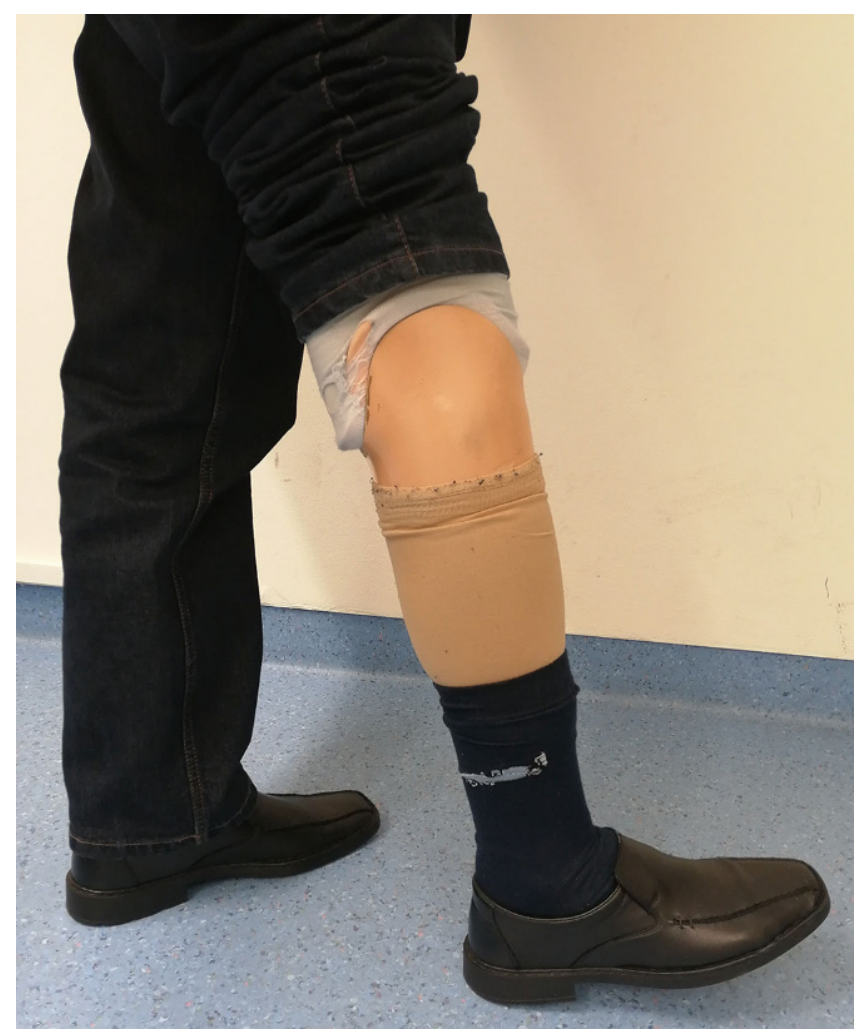

Abb. $8 \Delta$ Angelegte Exoprothese des rechten Unterschenkels bei Wiedervorstellung zur klinisch-radiologischen Verlaufskontrolle 3 Monate postoperativ mit voller Belastbarkeit des Stumpfes und flüssigem Gangbild manifestieren. Hierbei handelt es sich jedoch um eine absolute Rarität.

- Die marginale Resektion (R1-Status) eines TSGCT ist bei klinischer Symptomatik der therapeutische Goldstandard und führt in der Regel zu einer lokalen Tumorkontrolle. Dennoch sind Lokalrezidive vor allem beim diffusen Typ des TSGCT häufig.

- Die experimentelle intramedulläre Verbundosteosynthese der proximalen Tibia mit zwei kanülierten Schrauben und PMMA (Polymethylmethacrylat)-Knochenzement führte bei einem unterschenkelamputierten Patienten mit intraossärem TSGCT zur vollständigen Rehabilitation mit uneingeschränkter Exoprothesenversorgung und Wiedererlangung seiner Autonomie.

- Die PMMA-Augmentierung in diesem Fall weist neben dem Vorteil der vereinfachten nativradiologischen Rezidivdiagnostik möglicherweise einen positiven Effekt auf die lokale
Tumorkontrolle durch Thermodesinfektion auf.

\section{Korrespondenzadresse}

\section{Dr. med. Sebastian Klingebiel}

Klinik für Allgemeine Orthopädie und

Tumororthopädie, Universitätsklinikum Münster

Albert-Schweitzer Campus 1, 48149 Münster, Deutschland

sebastian.klingebiel@ukmuenster.de

Funding. Open Access funding provided by Projekt DEAL.

\section{Einhaltung ethischer Richtlinien}

Interessenkonflikt. S. Klingebiel, S. Mühl, W. Hartmann, K.N. Schneider, G. Gosheger, T.B. Budny, C. Rickert, D. Schorn, N. Deventer und T. Lübben geben an, dass kein Interessenkonflikt besteht.

Für diesen Beitrag wurden von den Autoren keine Studien an Menschen oder Tieren durchgeführt. Für die aufgeführten Studien gelten die jeweils dort angegebenen ethischen Richtlinien. Für Bildmaterial oder anderweitige Angaben innerhalb des Manuskripts, über die Patienten zu identifizieren sind, liegt von innen und/oder ihren gesetzlichen Vertretern eine schriftliche Einwilligung vor.

Open Access. Dieser Artikel wird unter der Creative Commons Namensnennung 4.0 International Lizenz veröffentlicht, welche die Nutzung, Vervielfältigung Bearbeitung, Verbreitung und Wiedergabe in jeglichem Medium und Format erlaubt, sofern Sie den/die ursprünglichen Autor(en) und die Quelle ordnungsgemäß nennen, einen Link zur Creative Commons Lizenz beifügen und angeben, ob Änderungen vorgenommen wurden.

Die in diesem Artikel enthaltenen Bilder und sonstiges Drittmaterial unterliegen ebenfalls der genannten Creative Commons Lizenz, sofern sich aus der Abbildungslegende nichts anderes ergibt. Sofern das betreffende Material nicht unter der genannten Creative Commons Lizenz steht und die betreffende Handlung nicht nach gesetzlichen Vorschriften erlaubt ist, ist für die oben aufgeführten Weiterverwendungen des Materials die Einwilligung des jeweiligen Rechteinhabers einzuholen.

Weitere Details zur Lizenz entnehmen Sie bitte de Lizenzinformation auf http://creativecommons.org/ licenses/by/4.0/deed.de. 


\section{Literatur}

1. Dal Cin P, Sciot R, Samson I, De Smet L, De Wever I, Van Damme B, Van den Berghe H (1994) Cytogenetic characterization of tenosynovial giant cell tumors (nodular tenosynovitis). Cancer Res 54:3986-3987

2. De Ponti A, Sansone V, Malcherè M (2003) Result of arthroscopic treatment of pigmented villonodular synovitis of the knee. Arthroscopy 19:602-607. https://doi.org/10.1016/s0749-8063(03)00127-0

3. Dundar A, Young JR, Wenger DE, Inwards $C Y$, Broski SM (2020) Unusual manifestations of diffuse-type tenosynovial giant cell tumor in two patients: importance of radiologic-pathologic correlation. Skeletal Radiol 49:483-489. https:// doi.org/10.1007/s00256-019-03325-7

4. Imakiire N, Fujino T, Morii T, Honya K, Mochizuki K, Satomi K, Fujioka Y (2011) Malignant pigmented Villonodular synovitis in the knee-report of a case with rapid clinical progression. Open Orthop J 5:13-16. https://doi.org/10. 2174/1874325001105010013

5. Jendrissek KA, Hotfiel T, Swoboda B, Söder S, Janka R (2016) Pigmentierte villonoduläre Synovialitis. Z Rheumatol 75:157-165. https://doi.org/ 10.1007/s00393-015-0028-4

6. Ma X, Shi G, Xia C, Liu H, He J, Jin W (2013) Pigmented villonodular synovitis: a retrospective study of seventy five cases (eighty one joints). Int Orthop 37:1165-1170. https://doi.org/10.1007/ s00264-013-1858-9

7. Myers BW, Masi AT (1980) Pigmented villonodular synovitis and tenosynovitis: a clinical epidemiologic study of 166 cases and literature review. Medicine 59:223-238

8. Safaee M, Oh T, Sun MZ, Parsa AT, McDermott MW, El-Sayed IH, Bloch O (2015) Pigmented villonodular synovitis of the temporomandibular joint with intracranial extension: a case series and systematic review. Head Neck 37:1213-1224. https://doi.org/ 10.1002/hed.23717

9. Stevenson JD, Jaiswal A, Gregory JJ, Mangham DC, Cribb G, Cool P (2013) Diffuse pigmented villonodular synovitis (diffuse-type giant cell tumour) of the foot and ankle. Bone Joint J 95B:384-390. https://doi.org/10.1302/0301-620X. 95B3.30192

10. West RB, Rubin BP, Miller MA, Subramanian $S$, Kaygusuz G, Montgomery K, Zhu S, Marinelli RJ, De Luca A, Downs-Kelly E, Goldblum JR, Corless CL, Brown PO, Gilks CB, Nielsen TO, Huntsman D, van de Rijn M (2006) A landscape effect in tenosynovial giant-cell tumorfromactivation ofCSF1 expression by a translocation in a minority of tumor cells. Proc Natl Acad Sci USA 103:690-695. https://doi.org/ 10.1073/pnas.0507321103

11. Xie G, Jiang N, Liang C, Zeng J, Chen Z, Xu Q, Qi R, Chen Y, Yu B (2015) Pigmented Villonodular Synovitis: a retrospective multicenter study of 237 cases. PLoS ONE. https://doi.org/10.1371/journal. pone. 0121451

Bureck, W., Kark, A., Gundelwein, I., Wendt, H., Behrendt, $M$. Langer, $M$.

\section{Schienenversorgung in der Handtherapie}

Bauanleitungen für statische, dynamische und statisch-progressive Schienen

Berlin Heidelberg: Springer-Verlag 2020, 1. Auflage, 202 S., über 300 Abb., (ISBN: 978-3-662-53787-9), Softcover + eBook: 59,99 EUR, eBook: 46,99 EUR

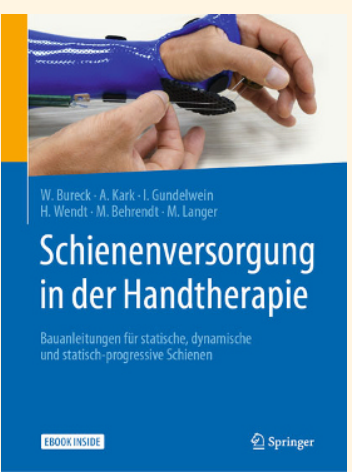

Das Buch "Schienenversorgung in der

Handtherapie" ist, wie der Titel andeutet, ein "klinisch" orientiertes Werk. Zeitgemäß wird ein Online-Zugang für das Buch vorgehalten.

Dieses Buch schließt eine relevante Lücke in der deutschsprachigen Literatur im Themengebiet der Handchirurgie/-therapie. Die ausgewiesenen und sehr erfahrenen Autorinnen und Autoren vermitteln eindrücklich die Inhalte. Das Layout ist zeitgemäß. Die großzügigen und farbigen Abbildungen vermitteln zusammen mit dem Text didaktisch ausgezeichnet die Kapitelinhalte.

Das Buch ist in 6 Sektionen unterteilt. Zunächst werden die Grundlagen des Schienenbaus, auch vor dem geschichtlichen Hintergrund, beleuchtet. Danach folgen die Herstellung statischer Schienen, Grundlagen, Grundwissen und Herstellung dynamischer Schienen, bevor das Themengebiet der Fertigschienen das Buch abrundet.

Das jeweilige Thema wird sorgfältig aufgearbeitet und die Autoren/-innen stellen die Inhalte fokussiert und anschaulich vor. Die Kapitel sind strukturiert aufgebaut und bestechen durch eindrückliche Abbildungen und Zeichnungen, die den praktischen Zugang zum Thema ermöglichen.
Das Buch "Schienenversorgung in der Handtherapie" ist ein essentieller Baustein im weitreichenden Themengebiet der Handchirurgie/-therapie. Es dient nicht nur zur Wissensvermittlung für Handtherapeuten/innen, sondern auch für Orthopädietechnik Mechaniker/-innen und als Nachschlagewerk für Ärzte/-innen, die letztlich diese Schienen "abnehmen" müssen und vor allem den Therapieverlauf überwachen sollen.

C. Spies (Bad Rappenau) 
Hier steht eine Anzeige.

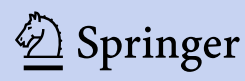

